\title{
Investigating the Tetraquark Structure of the New Mesons
}

\author{
M. Nielsen ${ }^{1}$, F. S. Navarra ${ }^{1}$, and M. E. Bracco ${ }^{2}$ \\ ${ }^{1}$ Instituto de Física, Universidade de São Paulo, C.P. 66318, 05389-970 São Paulo, SP, Brazil \\ ${ }^{2}$ Instituto de Física, Universidade do Estado do Rio de Janeiro, \\ Rua São Francisco Xavier 524, 20550-900 Rio de Janeiro, RJ, Brazil
}

\section{Received on 29 September, 2006}

\begin{abstract}
Using the QCD sum rule approach we investigate the vertex associated with the decay $D^{0}\left(0^{+}\right) \rightarrow D^{+} \pi^{-}$, where the scalar meson $D^{0}\left(0^{+}\right)$is considered as a four-quark state $(c d)(\bar{u} \bar{d})$. Although our results for the mass and partial decay width are smaller than the mass and the total decay width of the broad scalar meson $D_{0}^{* 0}(2308)$ reported by BELLE Collaboration, we can not discard the possibility that the BELLE's resonance can be interpreted as the four-quark state studied here.
\end{abstract}

Keywords: QCD sum rules; Four-quark states; Scalar mesons

The recent observations of the very narrow resonances $D_{s J}^{+}(2317)$ by BaBar [1], $D_{s J}^{+}(2460)$ by CLEO [2], and $X(3872)$ by BELLE [3], all of them with masses below quark model predictions, have stimulated a renewed interest in the spectroscopy of open charm and charmonium states. Due to their narrowness and small masses, these new mesons were considered as good candidates for four-quark states by many authors [4]. The idea of mesons as four-quark states is not new. Indeed, even Gell-Mann in his first work about quarks had mentioned that mesons could be made out of $(q \bar{q}),(q q \bar{q} \bar{q})$ etc. [5]. The best known example of applying the idea of four-quark states for mesons is for the light scalar mesons (the isoscalars $\sigma(500), f_{0}(980)$, the isodublet $\kappa(800)$ and the isovector $\left.a_{0}(980)\right)[6,7]$. In a four-quark scenario, the mass degeneracy of $f_{0}(980)$ and $a_{0}(980)$ is natural, the mass hierarchy pattern of the nonet is understandable, and it is easy to explain why $\sigma$ and $\kappa$ are broader than $f_{0}(980)$ and $a_{0}(980)$.

In refs. [8, 9] the method of QCD sum rules (QCDSR) [1012] was used to study the two-point functions for the mesons $D_{S J}^{+}(2317)$ and $X(3872)$ considering them as four-quark states in a diquark-antidiquark configuration. The results obtained for their masses are given in Table I.

Comparing the results in Table I with the resonance masses given by: $D_{s J}^{+}(2317)$ and $X(3872)$, we see that it is possible to reproduce the experimental value of the masses using a fourquark representation for these states.

The study of the three-point functions related to the decay widths $D_{s J}^{+}(2317) \rightarrow D_{s}^{+} \pi^{0}$ and $X(3872) \rightarrow J \psi \pi^{+} \pi^{-}$, using the diquark-antidiquark configuration for $D_{s J}$ and $X$, was done in refs. $[13,14]$. The results obtained for their partial decay widths are given in Table II.

From Table II we see that the partial decay width obtained in ref. [13], supposing that the mesons $D_{s J}^{+}(2317)$ is a fourquark state, is consistent with the experimental upper limit. However, in the case of the meson $X(3872)$, the partial decay width obtained in ref. [14] is much bigger than the experimen-

TABLE I: Numerical results for the resonance masses

\begin{tabular}{|c|c|c|}
\hline resonance & $D_{S J}$ & $X$ \\
\hline mass $(\mathrm{GeV})$ & $2.32 \pm 0.13$ & $3.93 \pm 0.15$ \\
\hline
\end{tabular}

TABLE II: Numerical results for the resonance decay widths

\begin{tabular}{|c|c|c|}
\hline decay & $D_{s J}^{+} \rightarrow D_{s}^{+} \pi^{0}$ & $X \rightarrow J / \psi \pi^{+} \pi^{-}$ \\
\hline$\Gamma(\mathrm{MeV})$ & $(6 \pm 2) \times 10^{-3}$ & $50 \pm 15$ \\
\hline$\Gamma_{\text {tot }}^{\text {exp }}(\mathrm{MeV})$ & $<5$ & $<2.3$ \\
\hline
\end{tabular}

tal upper limit to the total width.

In ref. [14] some arguments were presented to reduce the value of this decay width, by imposing that the initial fourquark state needs to have a non-trivial color structure. In this case, its partial decay width can be reduced to $\Gamma(X \rightarrow$ $\left.\left.J / \psi \pi^{+} \pi^{-}\right)\right)=(0.7 \pm 0.2) \mathrm{MeV}$. However, that procedure may appear somewhat unjustified and, therefore, more study is needed until one can arrive at a definitive conclusion about the structure of the meson $X(3872)$.

In ref. [8], besides the four-quark state $(c q)(\bar{s} \bar{q})$ representing the meson $D_{s J}^{+}(2317)$, it was also studied the configuration $(c q)(\bar{u} \bar{d})$ associated with a possible scalar meson that we will call $D\left(0^{+}\right)$(the $0^{+}$stands for $J^{P}$ ). The mass obtained for this state is: $m_{D\left(0^{+}\right)}=(2.22 \pm 0.21) \mathrm{MeV}$, in a very good agreement with the prediction made in ref. [15] for the $D\left(0^{+}\right)$ scalar meson: $m_{D\left(0^{+}\right)}=(2.215 \pm 0.002) \mathrm{MeV}$. This value was obtained in ref. [15] by supposing that the meson $D\left(0^{+}\right)$is the chiral partner of the meson $D$, with the same mass difference as the chiral pair $D_{S J}^{+}(2317)-D_{s}$. The authors of ref. [15] have also evaluated the decay widths $D_{s J}^{+} \rightarrow D_{s}^{+} \pi^{0}$ and $D\left(0^{+}\right) \rightarrow D \pi^{ \pm}$obtaining: $\Gamma\left(D_{s J}^{+} \rightarrow D_{s}^{+} \pi^{0}\right)=21.5 G_{A}^{2}$ $\mathrm{keV}$ and $\Gamma\left(D\left(0^{+}\right) \rightarrow D \pi^{ \pm}\right)=326 G_{A}^{2} \mathrm{MeV}$, where they expect $G_{A} \sim 1$.

Here, we extend the calculation done in refs. [8, 13] to study the vertex associated with the decay $D^{0}\left(0^{+}\right) \rightarrow D^{+} \pi^{-}$. The QCDSR calculation for the vertex, $D^{0}\left(0^{+}\right) D^{+} \pi^{-}$, centers around the three-point function given by

$$
T_{\mu}\left(p, p^{\prime}, q\right)=\int d^{4} x d^{4} y e^{i p^{\prime} \cdot x} e^{i q \cdot y}\left\langle 0\left|T\left[j_{D}(x) j_{5 \mu}(y) j_{0}^{\dagger}(0)\right]\right| 0\right\rangle,
$$

where $j_{0}$ is the interpolating field for the scalar $D^{0}\left(0^{+}\right)$meson 
[8]:

$$
j_{0}=\varepsilon_{a b c} \varepsilon_{d e c}\left[\left(d_{a}^{T} C \gamma_{5} c_{b}\right)\left(\bar{u}_{d} \gamma_{5} C \bar{d}_{e}^{T}\right)\right],
$$

where $a, b, c, \ldots$ are colour indices and $C$ is the charge conjugation matrix. In Eq. (1), $p=p^{\prime}+q$ and the interpolating fields for the $\pi^{-}$and $D^{+}$mesons are given by:

$$
j_{5 \mu}=\bar{u}_{a} \gamma_{\mu} \gamma_{5} d_{a}, \quad j_{D}=i \bar{d}_{a} \gamma_{5} c_{a} .
$$

The calculation of the phenomenological side proceeds by inserting intermediate states for $D, \pi$ and $D\left(0^{+}\right)$, and by using the definitions: $\left\langle 0\left|j_{5 \mu}\right| \pi(q)\right\rangle=i q_{\mu} F_{\pi},\left\langle 0\left|j_{D}\right| D\left(p^{\prime}\right)\right\rangle=\frac{m_{D}^{2} f_{D}}{m_{c}}$, $\left\langle 0\left|j_{0}\right| D\left(0^{+}\right)(p)\right\rangle=\lambda_{0}$. We obtain the following relation:

$$
T_{\mu}^{p h e n}\left(p, p^{\prime}, q\right)=\frac{\lambda_{0} m_{D}^{2} f_{D} F_{\pi} g_{D\left(0^{+}\right) D \pi} / m_{c}}{\left(p^{2}-m_{D\left(0^{+}\right)}^{2}\right)\left(p^{\prime 2}-m_{D}^{2}\right)\left(q^{2}-m_{\pi}^{2}\right)} q_{\mu}
$$

$$
\text { +continuum contribution , }
$$

where the coupling constant, $g_{D\left(0^{+}\right) D \pi}$, is defined by the onmass-shell matrix element: $\left\langle D \pi \mid D\left(0^{+}\right)\right\rangle=g_{D\left(0^{+}\right) D \pi}$. The con- tinuum contribution in Eq.(4) contains the contributions of all possible excited states.

In the case of the light scalar mesons, considered as diquark-antidiquark states, the study of their vertices functions using the QCD sum rule approach at the pion pole $[11,12,16]$, was done in ref.[17]. It was shown that the decay widths determined from the QCD sum rule calculation are consistent with existing experimental data. Here, we follow refs. $[13,17]$ and work at the pion pole. The main reason for working at the pion pole is that one does not have to deal with the complications associated with the extrapolation of the form factor [18]. The pion pole method consists in neglecting the pion mass in the denominator of Eq. (4) and working at $q^{2}=0$. In the OPE side one singles out the leading terms in the operator product expansion of Eq.(1) that match the $1 / q^{2}$ term. Since we are working at $q^{2}=0$, we take the limit $p^{2}=p^{\prime 2}$ and we apply a single Borel transformation to $p^{2}, p^{\prime 2} \rightarrow M^{2}$. In the phenomenological side, in the structure $q_{\mu}$ we get [13]:

$$
T^{p h e n}\left(M^{2}\right)=\frac{\lambda_{0} m_{D}^{2} f_{D} F_{\pi} g_{D\left(0^{+}\right) D \pi}}{m_{c}\left(m_{D\left(0^{+}\right)}^{2}-m_{D}^{2}\right)}\left(e^{-m_{D}^{2} / M^{2}}-e^{-m_{D\left(0^{+}\right)}^{2} / M^{2}}\right)+A e^{-s_{0} / M^{2}}+\int_{u_{0}}^{\infty} \rho_{c c}(u) e^{-u / M^{2}} d u,
$$

where $A$ and $\rho_{c c}(u)$ stands for the pole-continuum transitions and pure continuum contributions, with $s_{0}$ and $u_{0}$ being the continuum thresholds for $D\left(0^{+}\right)$and $D$ respectively. For simplicity, one assumes that the pure continuum contribution to the spectral density, $\rho_{c c}(u)$, is given by the result obtained in the OPE side. Therefore, one uses the ansatz: $\rho_{c c}(u)=\rho_{O P E}(u)$. In Eq.(5), $A$ is a parameter which, together with $g_{D\left(0^{+}\right) D \pi}$, has to be determined by the sum rule.

In the OPE side we single out the leading terms proportional to $q_{\mu} / q^{2}$. Transferring the pure continuum contribution to the OPE side, the sum rule for the coupling constant, up to dimension 7 , is given by:

$$
C\left(e^{-m_{D}^{2} / M^{2}}-e^{-m_{D\left(0^{+}\right)}^{2} / M^{2}}\right)+A e^{-s_{0} / M^{2}}=2\langle\bar{q} q\rangle\left[\frac{1}{2^{4} \pi^{2}} \int_{m_{c}^{2}}^{u_{0}} d u e^{-u / M^{2}} u\left(1-\frac{m_{c}^{2}}{u}\right)^{2}-\frac{m_{c}\langle\bar{q} q\rangle}{6} e^{-m_{c}^{2} / M^{2}}\right],
$$

with

$$
C=\frac{\lambda_{0} m_{D}^{2} f_{D} F_{\pi}}{m_{c}\left(m_{D\left(0^{+}\right)}^{2}-m_{D}^{2}\right)} g_{D\left(0^{+}\right) D \pi} .
$$

In the numerical analysis of the sum rules, the values used for the meson masses, quark masses and condensates are: $m_{D\left(0^{+}\right)}=2.2 \mathrm{GeV}, m_{D}=1.87 \mathrm{GeV}, m_{c}=1.2 \mathrm{GeV}$, $\langle\bar{q} q\rangle=-(0.23)^{3} \mathrm{GeV}^{3}$. For the meson decay constants we use $F_{\pi}=\sqrt{2} 93 \mathrm{MeV}$ and $f_{D}=0.20 \mathrm{GeV}$ [19]. We use $u_{0}=6 \mathrm{GeV}^{2}$ and for the current meson coupling, $\lambda_{0}$, we are going to use the result obtained from the two-point function in ref. [8]. Considering $2.6 \leq \sqrt{s_{0}} \leq 2.8 \mathrm{GeV}$ we get $\lambda_{0}=(3.3 \pm 0.3) \times 10^{-3} \mathrm{GeV}^{5}$.

In Fig. 1 we show, through the dots, the right-hand side (RHS) of Eq.(6) as a function of the Borel mass. We use the same Borel window as defined in ref.[8]. To determine $g_{D\left(0^{+}\right) D \pi}$ we fit the QCDSR results with the analytical expression in the left-hand side (LHS) of Eq.(6). Using $\sqrt{s_{0}}=2.7 \mathrm{GeV}$ we get: $C=1.25 \times 10^{-3} \mathrm{GeV}^{7}$ and $A=1.47 \times 10^{-3} \mathrm{GeV}^{7}$. Using the definition of $C$ in Eq.(7) and $\lambda_{0}=3.3 \times 10^{-3} \mathrm{GeV}^{5}$ (the value obtained for $\sqrt{s_{0}}=2.7 \mathrm{GeV}$ ) we get $g_{D\left(0^{+}\right) D \pi}=6.94 \mathrm{GeV}$. Allowing $s_{0}$ to vary in the interval $2.6 \leq \sqrt{s_{0}} \leq 2.8 \mathrm{GeV}$, the corresponding variation obtained for the coupling constant is $5 \mathrm{GeV} \leq g_{D\left(0^{+}\right) D \pi} \leq$ $7.5 \mathrm{GeV}$.

The coupling constant, $g_{D\left(0^{+}\right) D \pi}$, is related to the partial decay width through the relation:

$$
\Gamma\left(D^{0}\left(0^{+}\right) \rightarrow D^{+} \pi^{-}\right)
$$




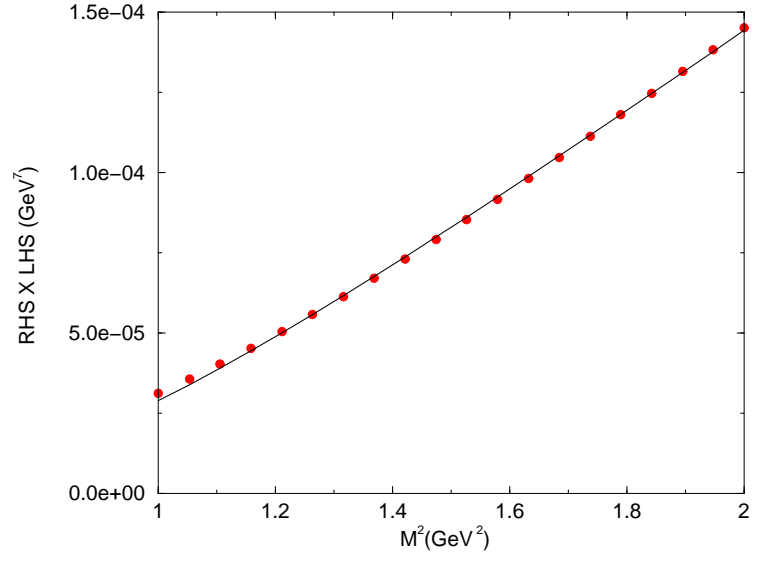

FIG. 1: Dots: the RHS of Eq.(6), as a function of the Borel mass. The solid line gives the fit of the QCDSR results through the LHS of Eq.(6).

$$
=\frac{1}{16 \pi m_{D\left(0^{+}\right)}^{3}} g_{D\left(0^{+}\right) D \pi}^{2} \sqrt{\lambda\left(m_{D\left(0^{+}\right)}^{2}, m_{D}^{2}, m_{\pi}^{2}\right)},
$$

where $\lambda(a, b, c)=a^{2}+b^{2}+c^{2}-2 a b-2 a c-2 b c$. Allowing $s_{0}$ to vary in the range discussed above we get:

$$
\Gamma\left(D^{0}\left(0^{+}\right) \rightarrow D^{+} \pi^{-}\right)=(120 \pm 20) \mathrm{MeV} .
$$

In Table III we show the partial decay width obtained in ref. [15], in ref. [13] and here for different decays. From the results in Table III we see that if one uses $G_{A}=0.6$, the result presented here and the result in ref. [13] are consistent with the results presented in ref. [15] for both decays.

Table III: Numerical results for the resonance partial decay widths from different approaches

\begin{tabular}{|c|c|c|c|}
\hline decay & ref. [15] & ref. [13] & this work \\
\hline$D_{s J}^{+} \rightarrow D_{S}^{+} \pi^{0}$ & $21.5 G_{A}^{2} \mathrm{keV}$ & $(6 \pm 2) \mathrm{keV}$ & \\
\hline$D^{0}\left(0^{+}\right) \rightarrow D^{+} \pi^{-}$ & $326 G_{A}^{2} \mathrm{MeV}$ & & $120 \pm 20 \mathrm{MeV}$ \\
\hline
\end{tabular}

It is important to notice that the BELLE Collaboration [20] has reported the observation of a rather broad scalar meson $D_{0}^{* 0}(2308)$ in the decay mode $D_{0}^{* 0}(2308) \rightarrow D^{+} \pi^{-}$with a total width $\Gamma \sim 270 \mathrm{MeV}$. Although both, the mass and the total decay width reported in [20], are bigger than the values found for the meson $D\left(0^{+}\right)$studied here, we can not discard the possibility that the BELLE's resonance can be interpreted as a four-quark state.

We have presented a QCD sum rule study of the vertex function associated with the strong decay $D^{0}\left(0^{+}\right) \rightarrow$ $D^{+} \pi^{-}$, where the scalar $D\left(0^{+}\right)$meson was considered as diquark-antidiquark state. We get for the partial decay width: $\Gamma\left(D^{0}\left(0^{+}\right) \rightarrow D^{+} \pi^{-}\right)=(120 \pm 20) \mathrm{MeV}$.

\section{Acknowledgements}

This work has been supported by CNPq and FAPESP.
[1] BABAR Coll., B. Auber et al., Phys. Rev. Lett. 90, 242001 (2003); Phys. Rev. D 69, 031101 (2004).

[2] CLEO Coll., D. Besson et al., Phys. Rev. D 68, 032002 (2003).

[3] BELLE Coll., S.-L. Choi et al., Phys. Rev. Lett. 91, 262001 (2003).

[4] for a review see E. S. Swanson, Phys. Rept. 429, 243 (2006).

[5] M. Gell-Mann, Phys. Lett. 8, 214 (1964).

[6] R. L. Jaffe, Phys. Rev. D 15, 267, 281 (1977); D 17, 1444 (1978).

[7] for a review see F.E. Close and N.A. Törnqvist, J. Phys. G 28, R249 (2002).

[8] M. E. Bracco, A. Lozea, R. D. Matheus, F. S. Navarra, and M. Nielsen, Phys. Lett. B 624, 217 (2005).

[9] R. Matheus, S. Narison, M. Nielsen, and J.-M. Richard, hep$\mathrm{ph} / 0608297$.

[10] M. A. Shifman, A. I. and Vainshtein, and V. I. Zakharov, Nucl. Phys., B 147, 385 (1979).

[11] L. J. Reinders, H. Rubinstein, and S. Yazaky, Phys. Rep. 127, 1 (1985).
[12] S. Narison, QCD spectral sum rules, World Sci. Lect. Notes Phys. 26, 1; QCD as a theory of hadrons, Cambridge Monogr. Part. Phys. Nucl. Phys. Cosmol. 17, 1-778 (2002) [hep$\mathrm{h} / 0205006]$

[13] M. Nielsen, Phys. Lett. B 634, 35 (2006).

[14] F. S. Navarra and M. Nielsen, Phys. Lett. B 639, 272 (2006).

[15] W. A. Bardeen, E. J. Eichten, C. T. Hill, Phys. Rev. D 68, 054024 (2003).

[16] S. Narison, Phys. Lett. B 175, 88 (1986); S. Narison and R. Tarrach, Phys. Lett. B 125, 217 (1983).

[17] T. V. Brito, F. S. Navarra, M. Nielsen, and M. E. Bracco, Phys. Lett. B 608, 69 (2005).

[18] R. S. Marques de Carvalho, F. S. Navarra, M. Nielsen, E. Ferreira, and H. G. Dosch, Phys. Rev. D 60, 034009 (1999).

[19] CLEO Coll., M. Artuso et al., Phys. Rev. Lett. 95, 251801 (2005).

[20] BELLE Coll., K. Abe et al., Phys. Rev. D 69, 112002 (2004). 